\title{
Unusual recurrent tongue spindle cell carcinoma with marked anaplasia occurring at the site of glossectomy for a well-differentiated squamous cell carcinoma: A case report
}

\author{
KOHEI OKUYAMA ${ }^{1}$, SHUICHI FUJITA ${ }^{2}$, SOUICHI YANAMOTO ${ }^{1}$, TOMOFUMI NARUSE ${ }^{1}$, YUKI SAKAMOTO ${ }^{1}$, \\ AKIKO KAWAKITA $^{1}$, KEISUKE OMORI $^{1}$, HIROKI TSUCHIHASHI ${ }^{1}$ and MASAHIRO UMEDA ${ }^{1}$ \\ Departments of ${ }^{1}$ Clinical Oral Oncology and ${ }^{2}$ Oral Pathology and Bone Metabolism, \\ Nagasaki University Graduate School of Biomedical Sciences, Nagasaki 852-8588, Japan
}

Received February 20, 2017; Accepted May 17, 2017

DOI: $10.3892 / \mathrm{mco} .2017 .1323$

\begin{abstract}
Spindle cell carcinoma (SpCC), which predominantly arises in the oral, pharyngeal and laryngeal mucosal tissues, is composed of a mixture of squamous and sarcomatoid components. The present study describes the case of a 62-year-old woman with SpCC recurrence 4 years after an initial surgery to remove a well-differentiated primary squamous cell carcinoma (SCC) of the tongue. The recurrent tumor was spherical and located deep within the tongue tissue, which differs from the typical manifestation of ulcerated masses of the mucosa. The majority of cases of recurrence involving $\mathrm{SpCC}$ are associated with radiotherapeutic treatment of the primary malignancy; however, the patient in the present study had not received postoperative radiotherapy for SCC. Furthermore, the recurrent tumor in the present case exhibited marked anaplasia and sarcomatoid features, and the absence of SCC elements upon biopsy rendered histological diagnosis difficult. In summary, the present findings suggest that immunohistochemical examination and identification of SCC components are essential for ensuring the accuracy of the histological diagnosis of recurrent $\mathrm{SpCC}$ following a primary epithelial malignancy.
\end{abstract}

\section{Introduction}

Spindle cell carcinomas (SpCCs) are biphasic tumors that typically exhibit a mesenchymal appearance and consist of

Correspondence to: Dr Kohei Okuyama, Department of Clinical Oral Oncology, Nagasaki University Graduate School of Biomedical Sciences, 1-7-1 Sakamoto, Nagasaki 852-8588, Japan

E-mail: okuyamak@nagasaki-u.ac.jp

Abbreviations: SpCC, spindle cell carcinoma; SCC, squamous cell carcinoma; CE-CT, contrast-enhanced computed tomography

Key words: spindle cell carcinoma, squamous cell carcinoma, epithelial-mesenchymal transition, cytokeratin AE1/AE3, vimentin squamous cell carcinoma (SCC) and malignant spindle cell components (1). SpCC preferentially occurs in the head and neck, particularly in the oral and laryngeal mucosal tissues. Macroscopically, these tumors are characterized by an ulcerated surface and polypoid appearance (1-6). Histological features associated with the transition area between spindle cells and SCC components/surface epithelium suggest an epithelial origin of the sarcomatoid component, which typically resembles fibrosarcoma or undifferentiated pleomorphic sarcoma (malignant fibrous histiocytoma) $(1,7,8)$. The development of SpCC is often associated with radiotherapy, and radiation-induced SpCC tends to include foci of osteosarcomatous, chondrosarcomatous, or rhabdomyosarcomatous differentiation $(1,9)$. In the present report, the authors discuss a patient with recurrent $\mathrm{SpCC}$ following glossectomy for a primary SCC. The tumor was spherical and located in the deep lingual layer of the tongue, and the patient had not undergone postoperative radiotherapy for the initial tumor, in contrast to patterns observed for typical cases of SpCC. As the mean interval between treatment of the primary tumor and local recurrence in cases of early-stage SCC of the tongue is $\sim 15$ months (10), the present case is also unusual in that recurrence was observed 4 years following initial glossectomy. The current report focuses on the peculiar features and the differential diagnosis of the present case.

\section{Case report}

In 2011, a 62-year-old woman with a history of sarcoidosis, psychotic depression and hypertension presented to the Department of Clinical Oral Oncology at Nagasaki University Hospital (Nagasaki, Japan) with reports of continuous stomatitis and pain on the left side of her tongue. Although the patient had smoked $\sim 10$ cigarettes per day for 20 years, she had been tobacco-free for 10 years. She also reported an alcohol intake of two glasses of shochu water per day. Intraoral examination revealed a presumably malignant tumor of the tongue, measuring $\sim 30 \times 28 \mathrm{~mm}$, which was immediately evaluated via imaging and incisional biopsy. Metastasis to two cervical lymph nodes on the affected side was suspected based on contrast-enhanced computed tomography (CE-CT) findings. 

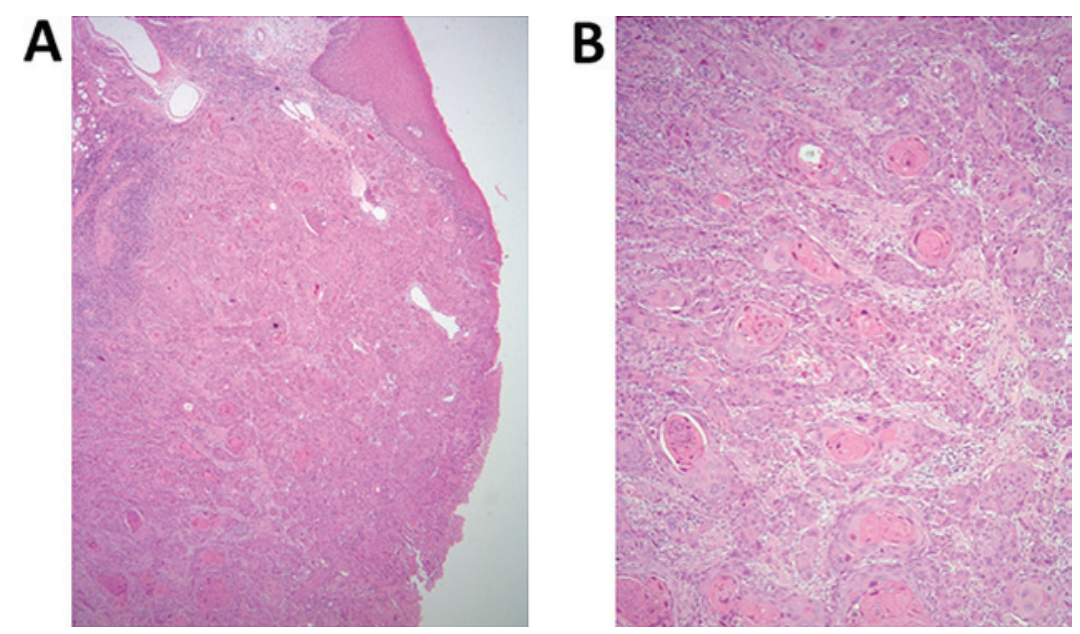

Figure 1. Biopsy specimen of the primary tumor. (A) Squamous cell carcinoma proliferation was present under the ulcerated lingual mucosa (magnification, x40; H\&E stain). (B) Cancer pearls were apparent in the tumor nests (magnification, x100; H\&E stain). H\&E, hematoxylin and eosin.
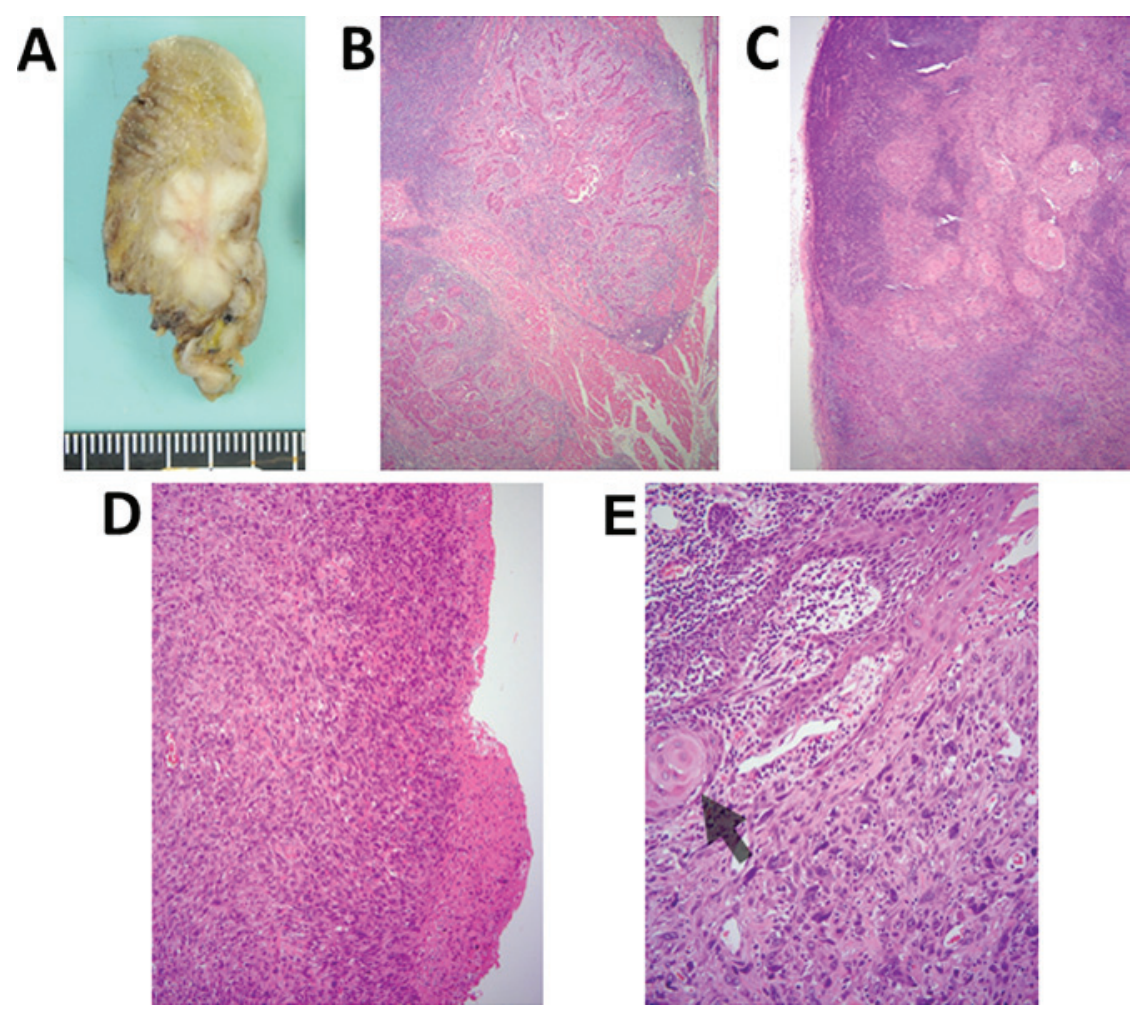

Figure 2. Resected primary tumor. (A) Analysis of surgical tissue indicated that the milky-white tumor had invaded the lingual muscle from the mucosa. (B) SCC-infiltrated striated muscle bundles accompanied by round cell infiltration was visible (magnification, x40; H\&E stain). (C) Lymphatic metastasis of SCC was observed at the level III neck region. No extranodal infiltration was detected (magnification, x40; H\&E stain). (D) A small degree of spindle cell proliferation was observed under the ulcer (magnification, x100; H\&E stain). (E) Atypical spindle cells with sarcomatous appearance had reached the dysplastic mucosal epithelium. SCC nests (arrow) were also observed (magnification, x200; H\&E stain). SCC, squamous cell carcinoma; H\&E, hematoxylin and eosin.

The biopsy specimen exhibited signs of well-differentiated SCC invading the submucosal tissue and lingual muscle from the mucosal epithelium, with apparent cancer pearls (Fig. 1A and B), following which the diagnosis of well-differentiated SCC of the left tongue (T2N2bM0, stage IV) was confirmed. Under general anesthesia, the patient underwent partial glossectomy with adequate tumor-free margins, left neck dissection, and soft tissue reconstruction using a vascularized forearm flap. As observed for the biopsy specimen, the tissue obtained during surgery was primarily indicative of SCC invading the lingual muscle (Fig. 2A-C). Notably, proliferation of atypical spindle cells with large hyperchromatic nuclei was observed beneath the ulcerative region of the tongue. These spindle cells transitioned out of the dysplastic mucosal epithelium at the periphery of the ulcer (Fig. 2D and E). Metastasis of SCC without extranodal infiltration was noted in one level III lymph node (left cervical). Atypical spindle cells were not detected in the metastatic focus. A histological diagnosis of 

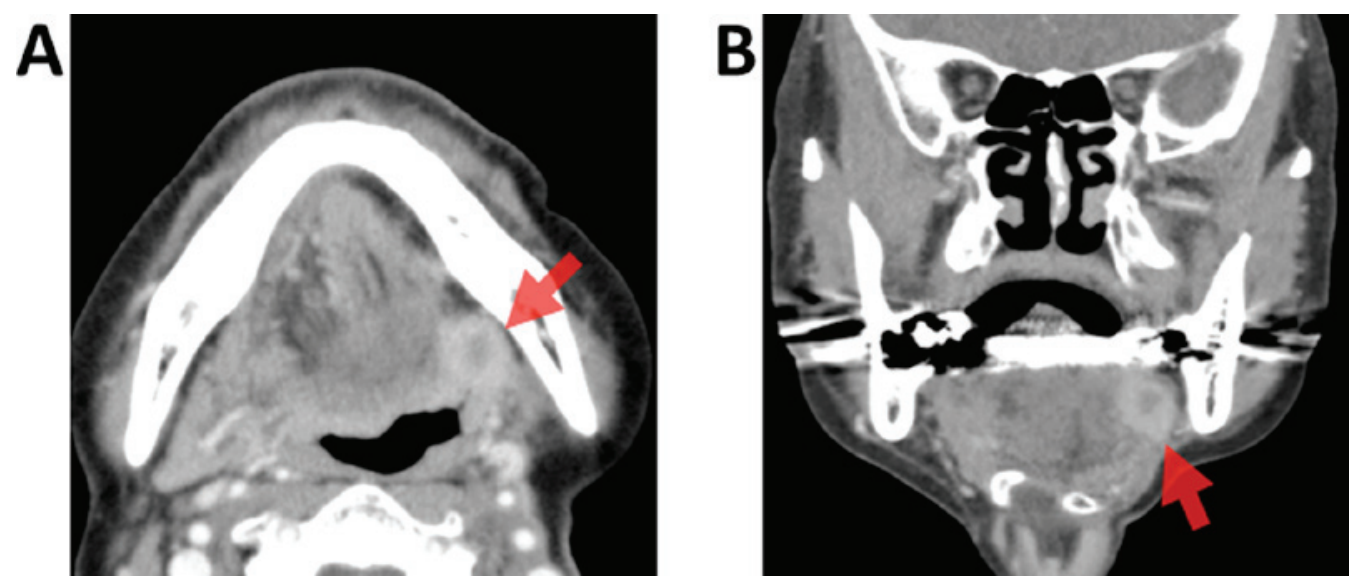

Figure 3. Recurrence of the tumor $(16 \times 13 \times 13 \mathrm{~mm})$ exhibiting heterogeneous enhancement at the root of the left tongue behind the grafted flap was suspected based on contrast-enhanced computed tomography findings (arrows). (A) Axial section; (B) coronal section.
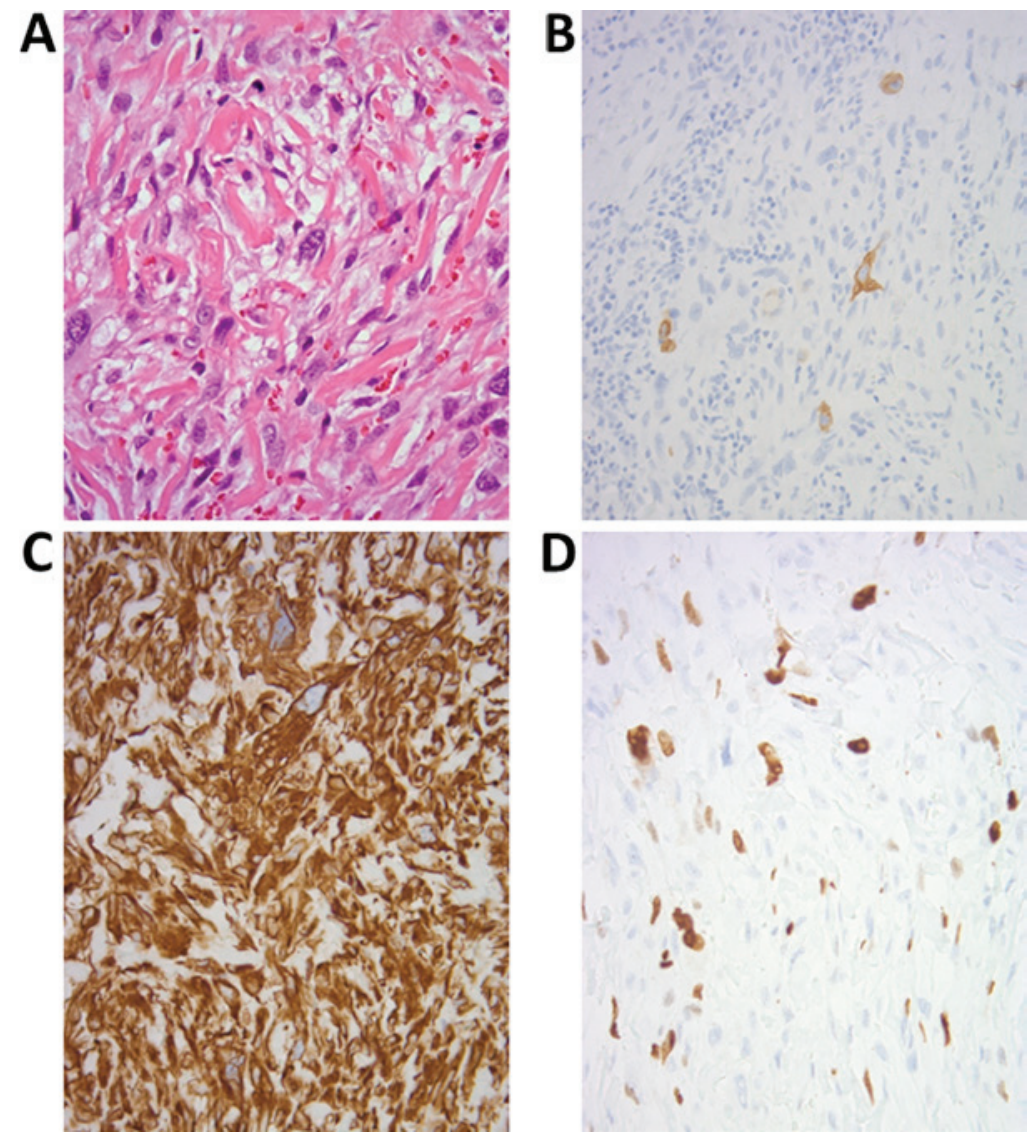

Figure 4. Incisional biopsy specimen from the secondary tumor. (A) Spindle cells included large or multiple nuclei with hyperchromatic change in the fibrous tissue (magnification, x400; hematoxylin and eosin stain). (B) Sparse atypical spindle cells were positive for cytokeratin AE1/AE3 (magnification, x200; IHC). (C) Vimentin was strongly expressed in the majority of spindle cells (magnification, x400; IHC). (D) The MIB-1 labeling index was $22.1 \%$ (magnification, $\mathrm{x} 400$; IHC). Hematoxylin (blue) was used as the counterstain for IHC. IHC, immunohistochemistry.

well-differentiated SCC was determined due to the relatively small number of spindle cells around the ulcerative region of the tongue.

As the tumor was $<1 \mathrm{~mm}$ from the margin of the surgical specimen, additional tissue was resected 1 month after the first surgery. The residual SCC was detected in the resected specimen, and there was no evidence of residual tumor around the resection margin. As the lymph node metastasis did not involve extracapsular spreading, postoperative adjuvant chemoradiotherapy was not performed.

During routine follow-up 4 years after the initial surgery, CE-CT revealed recurrence of the tumor $(16 \times 13 \times 13 \mathrm{~mm})$ at the root of the left tongue behind the grafted forearm flap, which exhibited heterogeneous enhancement. An incisional biopsy was performed under general anesthesia to confirm whether the tumor involved recurrent SCC or other independent 


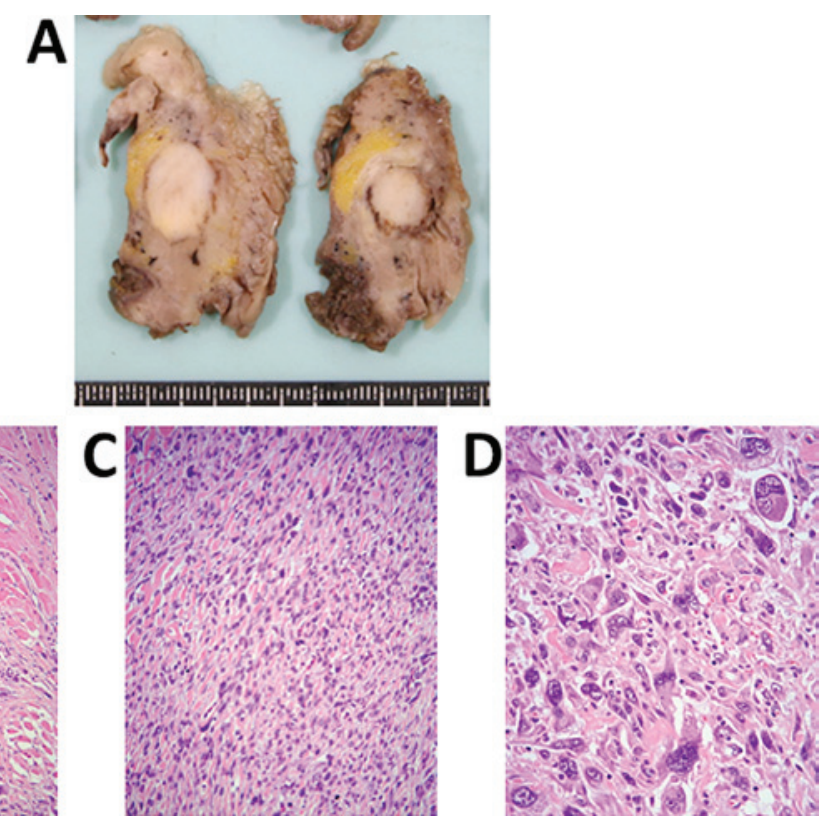

Figure 5. Surgically removed secondary tumor. (A) The cut surface revealed a spherical tumor, measuring $18 \mathrm{~mm}$ in maximum diameter, within the lingual muscle and adipose tissue. (B) Atypical tumor cells had infiltrated the striated muscle tissue (magnification, x200; H\&E stain). (C) Relatively uniform spindle cells with hyperchromatic nuclei proliferated within collagen bundles (magnification, x200; H\&E stain). (D) Anaplastic cells with bizarre and/or plural nuclei were observed (magnification, x400; H\&E stain). SCC, squamous cell carcinoma; H\&E, hematoxylin and eosin.
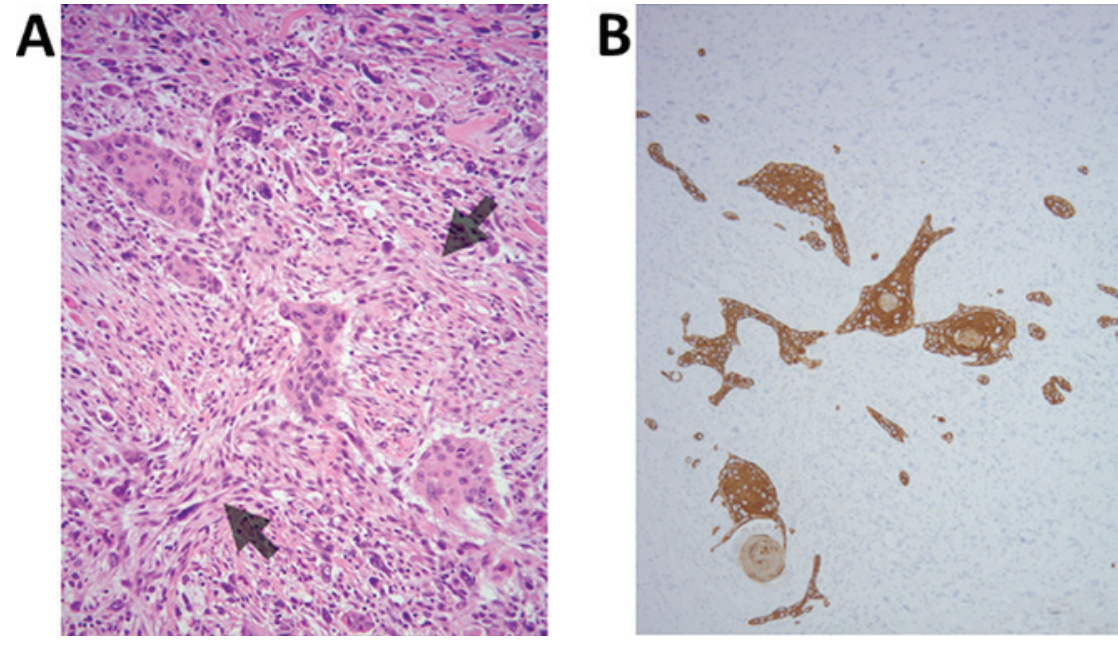

Figure 6. Surgically removed secondary tumor. (A) Storiform patterns of spindle cells (arrows) and residual SCC nests were observed (magnification, x200; hematoxylin and eosin stain). (B) Residual SCC and nearby scattered cells were positive for cytokeratin AE1/AE3 (magnification, x100; immunohistochemistry). SCC, squamous cell carcinoma.

malignancies, due to the location of the tumor deep within the tongue (Fig. 3A and B). Biopsy results revealed atypical spindle or oval cells with hyperchromatic abnormal nuclei accompanied by fibrous tissue, suggestive of sarcoma. No epithelial components were detected in the tumor (Fig. 4A and B). Immunohistochemically, cytokeratin AE1/AE3 was expressed in the sparse spindle cells, vimentin was strongly positive, and the MIB-1 (Ki-67) labeling index was 22.1\% (Fig. 4C-E), whereas the results for $\mathrm{S} 100$ and leukocyte common antigen (CD45) were negative. A preliminary diagnosis of sarcomatoid tumor was determined based on the biopsy results, as the possibility of a malignant epithelial tumor could not be ruled out due to the patient's history of primary SCC.
Under general anesthesia, left hemi-glossectomy, right neck dissection, and soft tissue reconstruction using a vascularized free rectus abdominis flap were performed using a mandibular swing approach due to the deep and posterior position of the tumor. The maximum diameter of the spherical tumor was $18 \mathrm{~mm}$, and it did not extend to the tongue mucosa (Fig. 5A). Microscopically, the tumor exhibited a sarcomatoid appearance without capsulation, and there was no extension to the mucosal epithelium; however, atypical spindle cells had infiltrated the adjacent striated muscle and adipose tissue (Fig. 5B). The tumor exhibited several sarcomatoid features: Monotonous atypical spindle cell proliferation in an ordered fashion (Fig. 5C); and intense anaplastic appearance involving 
cellular pleomorphism with large, bizarrely shaped nuclei or multinucleated cells (Fig. 5D). Furthermore, there was a malignant fibrous histiocytoma-like pattern, including storiform arrangement of collagen bundles (Fig. 6A). Finally, accurate examination using numerous tumor sections revealed the presence of tiny SCC components within the tumor (Fig. 6A). Morphological transition from SCC to sarcomatoid cells was not apparent. Immunohistochemically, the sarcomatoid cells were positive for vimentin and $\alpha$-smooth muscle actin, while AE1/AE3 was expressed in the SCC components and in sparse sarcomatoid cells (Fig. 6B). The two components were negative for S100, HMB-45, CD34, myoglobin and desmin. Therefore, we speculated that the sarcomatoid cells were derived from SCC due to their patchy immunoreactivity with AE1/AE3 and the patient's history of deeply invading SCC. Thus, a final diagnosis of SpCC was made.

The patient's postoperative course was event-free, with no evidence of tumor recurrence or metastasis at the 1-year follow-up.

The present study was conducted in accordance with the Declaration of Helsinki, and was approved by the Ethical Review Board of Nagasaki University. Appropriate consents, permissions, and releases were also obtained from the patient.

\section{Discussion}

SpCCs are biphasic, malignant epithelial tumors that consist of both SCC and sarcomatoid components. The SCC component may be scant or even inapparent on light microscopy (1). Therefore, histological diagnosis of SpCC is extremely difficult when SCC components are not detected (3). In such cases, there is a possibility for the tumor to be misdiagnosed as a fibrosarcoma or undifferentiated pleomorphic sarcoma (malignant fibrous histiocytoma). If sarcomatoid tissue includes features suggestive of differentiation, diagnoses of osteosarcoma, chondrosarcoma and rhabdomyosarcoma are also conceivable. Viswanathan et al (6) developed a diagnostic immunohistochemical algorithm for identifying spindle cell neoplasms in biopsied mucosa of the head and neck when the tumor lacks epithelial components. Nevertheless, diagnosis of SpCC remains difficult, as spindle cells of the SpCC demonstrate variable immunoreactivities with cytokeratin antibodies (2-4,7). Takata et al (11) suggested that the absence of staining for keratin in sarcomatoid tumor cells does not always exclude SpCC. In the present case, the biopsy specimen obtained from the secondary tumor lacked SCC components. However, SpCC was not eliminated as a possibility, based on the clinical course, small focus of spindle cell proliferation associated with mucosal epithelium in the primary carcinoma, and relatively low number of AE1/AE3-positive spindle cells. Once the preliminary diagnosis of sarcomatoid tumor was determined based upon the biopsy results, detailed examination of the resected tumor demonstrated a mixture of spindle cells and small SCC components. Thus, a final diagnosis of SpCC was determined. These findings suggest that SpCC should be considered during the differential diagnosis of recurrent sarcomatoid tumors occurring at the site of surgical resection of SCC.

SpCC typically exhibits exophytic or polypoid nodules and mucosal ulceration (1,3-6). However, in the present case, spherical nodules were observed within the deep muscle and adipose tissue of the tongue (Figs. 3 and 5A). Thus, we speculate that the present tumor developed from SCC persisting in the deep-infiltrating region following the patient's initial partial glossectomy. The sarcomatoid components may therefore have derived not from mucosal squamous epithelium but from the small number of SCC cells at the epithelial-mesenchymal transition (EMT).

Another peculiar feature of the present case is that the patient had not received radiotherapy following the partial glossectomy. The occurrence of SpCC is associated with smoking, alcohol consumption, and radiation exposure $(1,5,12)$, and instances of SpCC recurrence have been observed following radiotherapy for various primary malignancies, including SCC $(9,11,13)$. Radiation is thought to induce EMT in various normal and neoplastic tissues (14-17). Although SpCC in the present case was not induced by radiotherapy, the small focus of spindle cell elements transitioning from the dysplastic squamous epithelium in the primary tumor suggested that the neoplastic squamous cells exhibited induced characteristics of EMT. A number of reports have also suggested that various forms of sarcoma, such as malignant fibrous histiocytoma (undifferentiated pleomorphic sarcoma), can manifest as locoregional recurrence of SCC following radiotherapy of the head and neck (18-21). We speculate that some sarcomas may emerge as sarcomatoid components of SpCC. However, if the number of SCC components is extremely small, the tumor may be misdiagnosed as SpCC. Thus, the identification of epithelial components is required for the differential diagnosis of SpCC in cases of recurrent radiation-induced sarcoma at the site of previous surgical resection of a malignant epithelial tumor.

In conclusion, SpCC with marked anaplasia remains difficult to diagnose, as noted in the present case. The present findings further suggest that, during histological diagnosis of recurrent sarcomatoid tumors associated with primary epithelial malignancies, immunohistochemical examination and identification of SCC components are essential for ensuring the accuracy of the diagnosis.

\section{References}

1. Cardesa A and Zidar N: Spindle cell carcinoma. In: World Health Organization Classification of Tumours, Pathology \& Genetics, Head and Neck Tumours. Barnes L, Eveson J, Reichart P, Sidransky D (eds). IARCPress, Lyon, pp127-128, 2005.

2. Biradar MV, Dantkale SS, Abhange RS, Kamra HT and Birla K: Spindle cell carcinoma of the tongue: A rare variant of squamous cell carcinoma. Ecancermedicalscience 8: 447, 2014

3. Rizzardi C, Frezzini C, Maglione M, Tirelli G and Melato M: A look at the biology of spindle cell squamous carcinoma of the oral cavity: Report of a case. J Oral Maxillofac Surg 61: 264-268, 2003.

4. Romañach MJ, Azevedo RS, Carlos R, de Almeida OP and Pires FR: Clinicopathological and immunohistochemical features of oral spindle cell carcinoma. J Oral Pathol Med 39: 335-341, 2010.

5. Thompson LD, Wieneke JA, Miettinen M and Heffner DK: Spindle cell (sarcomatoid) carcinomas of the larynx: A clinicopathologic study of 187 cases. Am J Surg Pathol 26: 153-170, 2002.

6. Viswanathan S, Rahman K, Pallavi S, Sachin J, Patil A, Chaturvedi P, D'Cruz A, Agarwal J and Kane SV: Sarcomatoid (spindle cell) carcinoma of the head and neck mucosal region: A clinicopathologic review of 103 cases from a tertiary referral cancer centre. Head Neck Pathol 4: 265-275, 2010. 
7. Bavle RM, Govinda G, Venkataramanaiah PG, Muniswamappa S and Venugopal R: Fallacious carcinoma- Spindle cell variant of squamous cell carcinoma. J Clin Diagn Res 10: ZD05-ZD08, 2016.

8. Watson RF, Chernock RD, Wang X, Liu W, Ma XJ, Luo Y, Wang H, El-Mofty SK and Lewis JS Jr: Spindle cell carcinomas of the head and neck rarely harbor transcriptionally-active human papillomavirus. Head Neck Pathol 7: 250-257, 2013.

9. Leifer C, Miller AS, Putong PB and Min BH: Spindle-cell carcinoma of the oral mucosa. A light and electron microscopic study of apparent sarcomatous metastasis to cervical lymph nodes. Cancer 34: 597-605, 1974.

10. Pinsolle V, Truilhé Y, Majoufre C, Michelet V and Pinsolle J: Posterior marginal mandibulectomy for cancer of the oral cavity and otopharynx. Experience with 14 clinical cases. Ann Chir Plast Esthet 42: 223-227, 1997.

11. Takata T, Ito H, Ogawa I, Miyauchi M, Ijuhin $\mathrm{N}$ and Nikai $\mathrm{H}$ Spindle cell squamous carcinoma of the oral region. An immunohistochemical and ultrastructural study on the histogenesis and differential diagnosis with a clinicopathological analysis of six cases. Virchows Arch A Pathol Anat Histopathol 419: 177-282, 1991

12. Ramamurti A, Venkataraman M, Narasimhan M and Rao SR: Spindle cell carcinoma of the gingiva: A rare occurrence. Contemp Clin Dent 4: 500-503, 2013.

13. Lichtiger B, Mackay B and Tessmer CF: Spindle-cell variant of squamous carcinoma. A light and electron microscopic study of 13 cases. Cancer 26: 1311-1320, 1970.

14. Jung JW, Hwang SY, Hwang JS, Oh ES, Park S and Han IO: Ionising radiation induces changes associated with epithelial-mesenchymal transdifferentiation and increased cell motility of A549 lung epithelial cells. Eur J Cancer 43: 1214-1224, 2007.
15. Pan Y, Zhou C, Yuan D, Zhang J and Shao C: Radiation exposure promotes hepatocarcinoma cell invasion through epithelial mesenchymal transition mediation by $\mathrm{H} 2 \mathrm{~S} / \mathrm{CSE}$ pathway. Radiat Res 185: 96-105, 2016.

16. Zhang H, Luo H, Jiang Z, Yue J, Hou Q, Xie R and Wu S: Fractionated irradiation-induced EMT-like phenotype conferred radioresistance in esophageal squamous cell carcinoma. J Radiat Res 57: 370-380, 2016.

17. Zhou YC, Liu JY, Li J, Zhang J, Xu YQ, Zhang HW, Qiu LB, Ding GR, Su XM, Mei-Shi and Guo GZ: Ionizing radiation promotes migration and invasion of cancer cells through transforming growth factor-beta-mediated epithelial-mesenchymal transition. Int J Radiat Oncol Biol Phys 81: 1530-1537, 2011.

18. Makimoto Y, Yamamoto S, Takano H, Motoori K, Ueda T, Kazama T, Kaneoya K, Shimofusa R, Uno T, Ito H, et al: Imaging findings of radiation-induced sarcoma of the head and neck. Br J Radiol 80: 790-797, 2007.

19. Marchitto G, Marci V, Berrone M and Pentenero M: Early arising sarcoma after adjuvant radiotherapy for oral squamous cell carcinoma. J Oral Maxillofac Surg 74: 862.e1-e8, 2016.

20. Sadati KS, Haber M and Sataloff RT: Malignant fibrous histiocytoma of the head and neck after radiation for squamous cell carcinoma. Ear Nose Throat J 83: 278, 280-281, 2004.

21. Satomi T, Watanabe M, Kaneko T, Matsubayashi J, Nagao T and Chiba H: Radiation-induced malignant fibrous histiocytoma of the maxilla. Odontology 99: 203-208, 2011. 